Plasma Medicine, 6(1): v-vi (2016)

\title{
Preface: Bioplasmas and Plasmas with Liquids
}

The Bioplasma and Plasmas with Liquids joint conference of the COST Actions TD1208 Electrical Discharges with Liquids for Future Applications and MP1101 Biomedical Applications of Atmospheric Pressure Plasma Technology was held in Bertinoro, Italy on September 13-16, 2015.

The purpose of the Bioplasma and Plasmas with Liquids joint conference was to bring together researchers of different fields, and to establish an open forum for presentation and discussion of the latest advances in the fields of electrical discharges with liquids and plasma medicine, by bridging the scientific communities associated with the COST Actions TD1208 Electrical Discharges with Liquids for Future Applications and MP1101 Biomedical Applications of Atmospheric Pressure Plasma Technology.

The topics discussed during the meeting encompassed: plasma sources for biomedical applications; bio-plasma interactions; functional coatings of biomaterials; plasma therapeutics; plasmas generated directly in the liquid phase; atmospheric plasmas interacting with liquids; elementary physical and chemical processes initiated in liquid phase by electrical discharges; interaction of plasma reactive species with materials and surfaces; applications in water treatment and advanced oxidation processes; biomedical applications; applications in surface treatment and in nanosciences; and organic chemistry applications with their related phenomena. This range of topics represents an excellent overview of the state of art in bioplasma and interactions of plasmas with liquids.

This special issue of Plasma Medicine gathers selected papers from regular and invited contributions held at the Bioplasmas and Plasmas with Liquids joint conference; each contribution submitted for publication has been peer reviewed following the standard procedures of the journal. The editors are very grateful to all the referees for their careful support in improving the original manuscripts.

At the end, 9 manuscripts were accepted for publication, covering a wide spectrum of topics, including: plasma assisted disinfection and cancer treatment; nanomaterial synthesis; plasma treatment of liquids and their chemical characterization; and theoretical and experimental studies on sources where the plasma is generated in water or is in contact with water. It is an honour to present this Special Issue of the Plasma Medicine journal and we deeply thank the authors for their contribution.

Finally, we would like to thank the attendees and invited speakers, the members of the international scientific committee, the local organizing committee, the organizing secretariat and the conference sponsors that provided financial support. All were essential to the success of the joint conference. The greatest thanks are devoted to the COST Association for financial support of the conference participants under both COST Actions. 
Guest Editors:

Dr. Matteo Gherardi

Università di Bologna

Bologna, Italy

Prof. Vittorio Colombo

Università di Bologna

Bologna, Italy

Prof. Frantisek Krčma

Brno University of Technology

Brno, Czech Republic

Prof. Miles Turner

Dublin City University

Dublin, Ireland 